\title{
Heredity of Structure Inhomogeneity under Phase Transformations in Textured Zr-20\%Nb Alloy
}

\author{
Yu. Perlovich, H.J. Bunge*, M. Isaenkova and V. Fesenko \\ Moscow Engineering Physics Institute, Kashirskoe shosse 31, 115409 Moscow, Russia \\ * Institut für Metallkunde und Metallphysik, TU Clausthal, Grosser Bruch 23, \\ 38678 Clausthal-Zellerfeld, Germany
}

\begin{abstract}
Development of $\beta \rightarrow \infty \omega$ and $\beta \rightarrow>\alpha$ phase transformations in the rolled quenched alloy $\mathrm{Zr}-20 \% \mathrm{Nb}$ was studied by methods of X-ray texture diffractometry. Usage of a position sensitive detector allowed to construct, besides usual texture pole figures, distributions of X-ray line broadening and lattice elastic deformation depending on the orientation of reflecting planes. Diagrams of correlation between pole figures of different types for $\beta$-, $\omega$ - and $\alpha$-phases were analyzed to reveal factors controlling inhomogeneity of phase transformations and substructure features of derivative phases.
\end{abstract}

\section{INTRODUCTION}

All rolled metal materials exhibit a sharp substructure inhomogeneity, which develops along with formation of the rolling texture and reflects the inhomogeneous distribution of residual deformation effects in grains with different orientations. According to [1-2], the main features of this distribution are the following: 1) grain dispersion and lattice distortion are maximal in texture minima and minimal in texture maxima; 2) texture maxima are split into regions with elastic stresses of opposite signs. Hence, the specific stored energy depends on grain orientation and increases at slopes of texture maxima. If the deformed material suffers the phase transformation, the question arises of whether the derivative phases inherit the substructure inhomogeneity of the original phase or some equalizing of lattice conditions happens.

This question can be answered now on the basis of experimental results obtained by the new X-ray diffractometric technique using a position sensitive detector (PSD) and automated data treatment [3-5]. In the course of PSD texture measurements the X-ray line (hki) profile is registered now for each normal $<\mathrm{hkl}>$ orientation, and its physical broadening and peak position are determined by use of the approximation fitting procedure. By analogy with the integral intensity pole figure, i.e.the usual texture pole figure (TPF), the half-width pole figure (WPF) and the peak position pole figure (PPF) are constructed. Taking into account that the half-width of the X-ray line characterizes the grain dispersion and the lattice distortion along the normal to the reflecting plane [6], whilst the peak position depends on the value and the sign of elastic stresses acting along the same normal, these three pole figures describe in details the substructure of the phase of interest as well as its inhomogeneity. As applied to studies in phase transformations (PT), this approach opens quite new possibilities to track the PT development in the grains with definite orientations as well as to observe the structural rearrangement along definite directions.

The diffusionless. $\beta->\omega$ phase transformation in the quenched cold-rolled $\mathrm{Zr}-20 \% \mathrm{Nb}$ alloy was investigated using the texture methods of X-ray PSD diffractometry. The process was split into subprocesses located in grains with different orientations, lattice conditions and elastic stresses. Though the problem of the martensite-like $\omega$-phase in $\mathrm{Zr}$ - and Ti-alloys is studied for many years [7], some of its aspects require additional elucidation concerning, in particular, the role of elastic stresses and $\beta$-lattice distortion. In order to reveal the characteristic features of the PT $\beta \rightarrow \infty$, it was compared with the PT $\beta \rightarrow \alpha$, which develops in the similar sample at the higher temperature. 


\section{MATERIAL AND THE EXPERIMENTAL PROCEDURE}

The alloy $\mathrm{Zr}-20 \% \mathrm{Nb}$ has the monotectoid composition and is convenient for modelling of different PT processes in $\mathrm{Zr}$ alloys. At the temperature $610^{\circ} \mathrm{C}$ the alloy experiences $\mathrm{PT} \beta \leftrightarrow->\alpha$, where the high-temperature $\beta$-phase has the BCC crystalline lattice and the low-temperature $\alpha$-phase has the HCP lattice. These phases are connected by the Burgers orientation relationship:

$$
\left.\{011\}_{\beta}\left\|(0001)_{\alpha},\langle 111\rangle_{\beta}\right\|<11 \overline{2} 0\right\rangle_{\alpha} \text {. }
$$

The metastable $\omega$-phase forms either by quenching or by annealing of quenched alloy. Similar to the $\alpha$-phase, the $\omega$-phase has a hexagonal lattice; but the ratio c/a for these phases differs significantly: $(\mathrm{c} / \mathrm{a})_{\alpha}=1.593$, while $(\mathrm{c} / \mathrm{a})_{\mathrm{w}}=0.617$. PT $\beta \rightarrow \omega$ obeys the following orientation relationship [8]:

$$
\left.\left.\{111\}_{\beta} \|(0001)_{\omega},<011\right\rangle_{\beta} \|<11 \overline{2} 0\right\rangle_{\omega} \text {. }
$$

Quenching of a forged cast from $1000^{\circ} \mathrm{C}$ provided retaining of the $\beta$-phase at room temperature, while its subsequent cold rolling up to a deformation degree of $98 \%$ resulted in formation in the quenched phase of the rolling texture accompanied by development of the inhomogeneous substructure. The obtained $60 \mu \mathrm{m}$ thick foil was annealed in vacuum at $400^{\circ} \mathrm{C}$ to cause $\beta \rightarrow \infty \quad$ PT and at $500^{\circ} \mathrm{C}$ to cause $\beta \rightarrow \alpha$ PT Duration of used annealings varied from $0.25 \mathrm{~h}$ to $10 \mathrm{~h}$.

The presented results were obtained by use of the SIEMENS X-ray texture diffractometer D500/TX equipped with a PSD and a multichannel analyser. The simultaneous viewing angle of the PSD is $9^{\circ}$ and the angular resolving power was chosen $0.05^{\circ}$. The treatment of diffraction spectra measured for 1009 sample orientations provided construction of texture pole figures (TPF), half-width pole figures (WPF) and peak position pole figures (PPF) for $\beta-, \omega-$ and $\alpha$-phases. The angular radius of obtained pole figures is $70^{\circ}$. TPFs are constructed in units of pole density and WPFs - in angular units. PPFs are recalculated in such a manner, that they display the spatial distribution of relative deviations of the interplanar spacing $\Delta \mathrm{d} / \mathrm{d}$ from the weighted average value on a percentage basis. Depending on the deviation sign ("+" or "-"), elastic extension or compression of the crystalline lattice with respect to its average condition takes place along the direction in question. The used X-ray method and the principal features of data treatment are described in more detail in $[2,3]$.

The correlation between different pole figures is analyzed by use of correlation diagrams, where the values of the respective points in considered pole figures are plotted against each other. In order to distinguish tendencies corresponding to different texture maxima, points of different types were used in these diagrams. Since the pole figures $\{111\}_{\beta}$ and $(0001)_{\omega}$ as well as $\{011\}_{\beta}$ and $(0001)_{\alpha}$ are connected in pairs according to the foregoing orientation relationships, namely these pole figures are compared to analyze regularities of corresponding phase transformations. By measurement of the indicated pole figures, the X-ray lines $(222)_{\beta},(0002)_{\omega},(022)_{\beta}$ and $(0004)_{\alpha}$ were used, respectively.

\section{MAIN RESULTS}

Fig.1 presents incomplete TPF $\{111\}, \mathrm{WPF}(222)$ and $\mathrm{PPF}(222)$ for the $\beta-\mathrm{Zr}$ phase, while Fig.2 TPF(0001), WPF(0002) and PPF(0002) for the $\omega$-phase. The rolling texture of $\beta-Z r$ consists of components $\{001-113\}<011>$ and $\{111\}<011>$, so that the TPF $\{111\}_{\beta}$ in Fig. 1-a contains four maxima of the first component at the circle with an angular radius of $55^{\circ}$ and one maximum of the second component at the centre. Maxima of the component $\{001-113\}<011>$ differ in their positions relative to $R D$ and TD, being situated in the zones of different residual elastic deformations. Therefore, by subsequent construction of correlation diagrams we distinguish points belonging to maxima situated at different diameters of pole figure. as well as at its centre. The mutually associated pole figures for $\beta$ - and $\alpha$-phases, constructed on the basis of texture measurements by use of X-ray lines $(011)_{\beta}$ and $(0004)_{\alpha}$ respectively, were presented in $[4,5]$. In TPF $\{011\}_{\beta}$ four extended maxima of the main component $\{001-113\}<011>$ have quite identical positions at a distance of $45^{\circ}$ from the centre, unlike TPF $\{111\}_{\beta^{\circ}}$ Therefore, points of only one type correspond to this texture component in the correlation diagrams.

In Fig. 3 the diagrams are plotted to reveal the character of correlation between TPF $(0001)_{\omega}$ and $\operatorname{TPF}\{111\}_{\beta}$ (a) as well as between $\operatorname{TPF}(0001)_{\alpha}$ and $\operatorname{TPF}\{011\}_{\beta}$ (b). When constructing correlation diagrams, measured integral intensities were recalculated into relative intensities, so that the maximal 


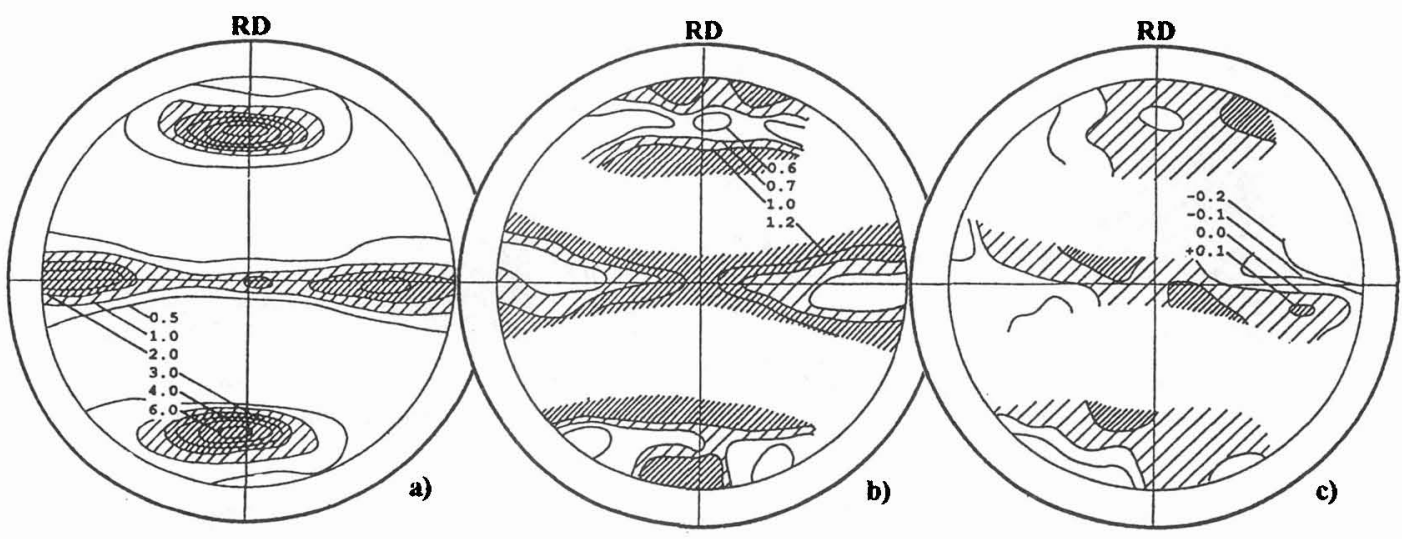

Figure 1: Pole figures for $\beta-Z r$ phase: a) TPF $\{111\}$; b) WPF(222); c) $\operatorname{PPF}(222)$.

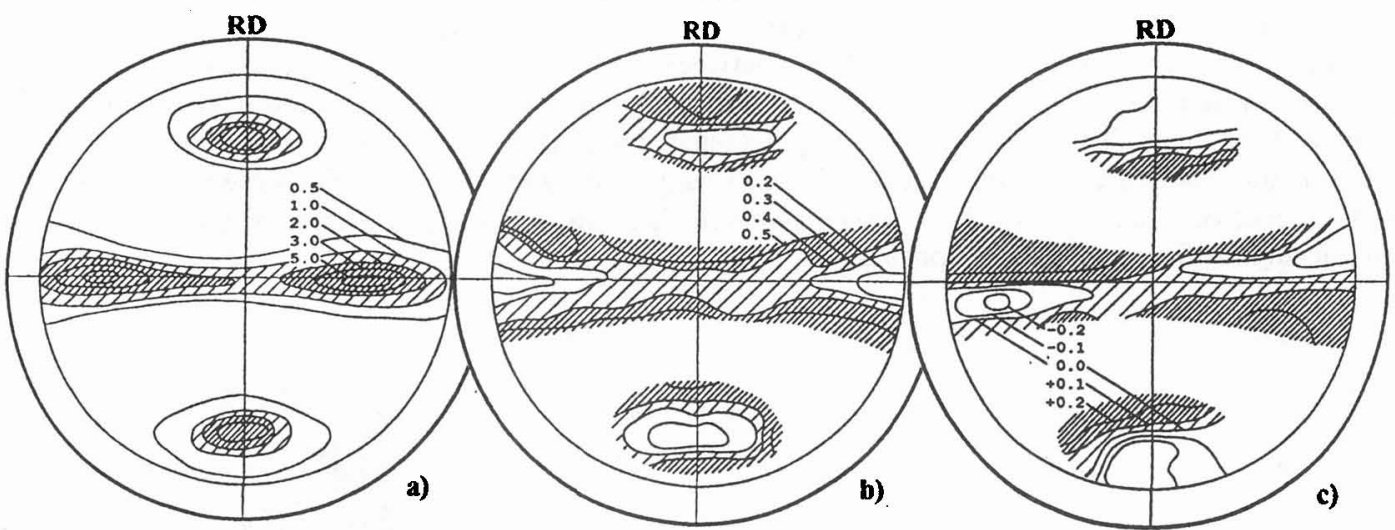

Figure 2: Pole figures for $\omega$-phase: a) TPF(0001); b) WPF(0002); c) PPF(0002).

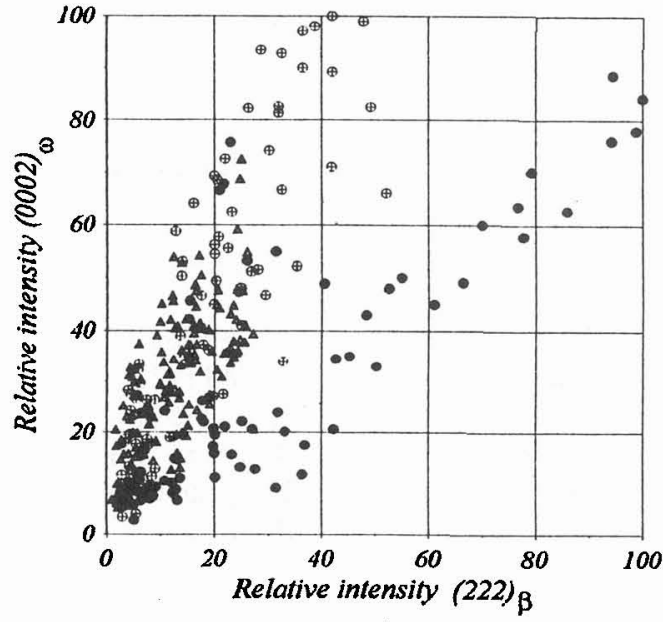

a)

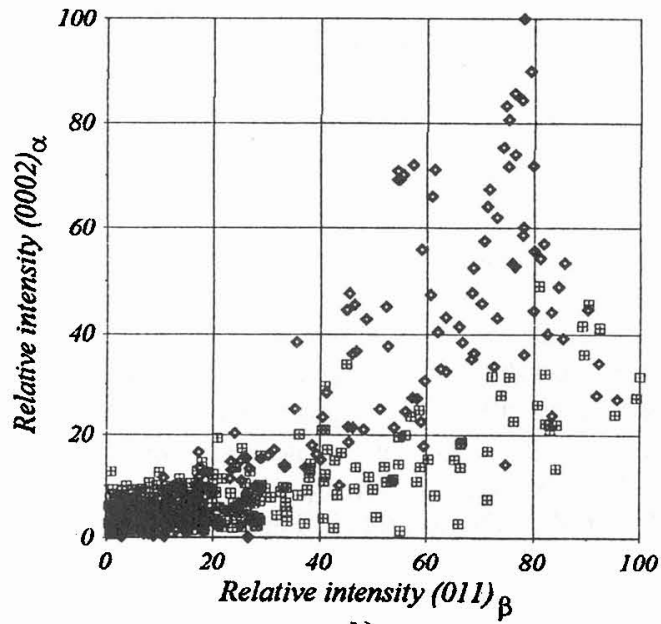

b)

Figure 3: Correlation betwecn: a) $\operatorname{TPF}(0001)_{\omega}$ and $\operatorname{TPF}\{111\}_{\beta}$; b) $\operatorname{TPF}(0001)_{\alpha}$ and $\operatorname{TPF}\{011\}_{\beta}$. TPFs $\{111\}_{\beta}$ and (0001) $\div \bullet \bullet$ - maxima at the diameter RD-RD; $\oplus-$ maxima at the diameter TD-TD; $\Delta$ - central maximum ; TPFs $\{011\}_{\beta}$ and $(0001)_{\alpha}^{\omega}$ : $:$ - maxima at a distance of $45^{\circ}$ from the centre; $\diamond$ - maxima at the diameter RD-RD. 


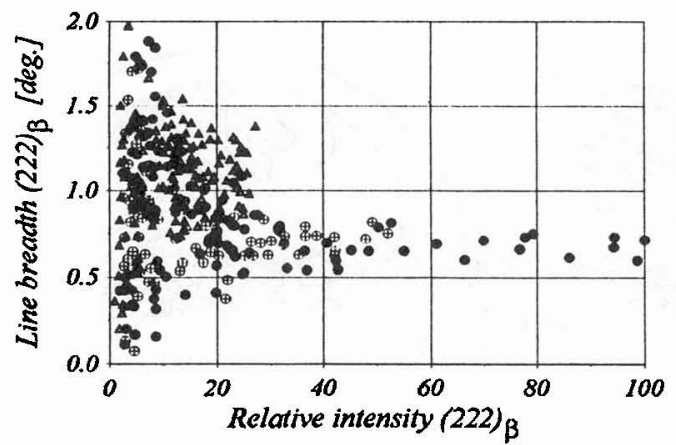

a)

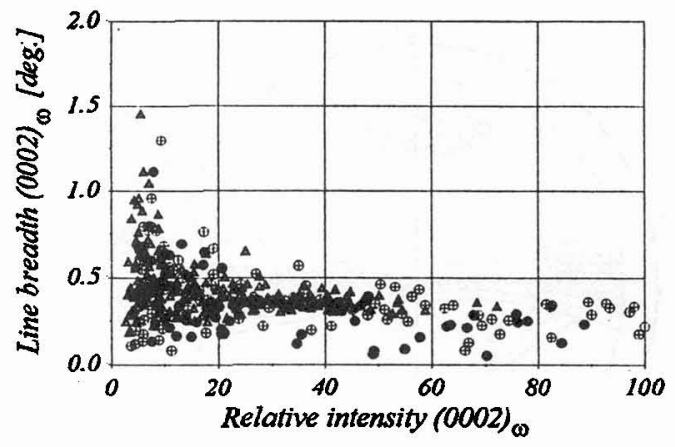

b)

Figurc 4: Correlation bctween: a) $\operatorname{WPF}(222)_{\beta}$ and $\operatorname{TPF}\{111\}_{\beta} ;$ b) $\left.\operatorname{WPF}_{(0002}\right)_{\omega}$ and $\operatorname{TPF}(0001)_{\omega}$

value of integral intensity for the corresponding pole figure was taken equal to 100 . Deviations of experimental points from the diagram diagonal should be considered as a manifestation of the fact, that different equivalent variants of the same orientation relationship realizes with unequal probabilities. Diagrams in Fig. 4 characterize the correlation between WPF $(222)_{\beta}$ and TPF $\{111\}_{\beta}(a)$ as well as between $\mathrm{WPF}(0002)_{\omega}$ and $\mathrm{TPF}(0001)_{\omega}(\mathrm{b})$, i.e. between lattice condition and volume fraction of $\beta$ - and $\omega$-grains, estimated by X-ray lines (222) $)_{\beta}$ and $(0002)_{\omega}$. Comparison of these diagrams allow to reveal features of substructure changes connected with PT $\beta->\omega$. Changes of lattice condition along the same direction in consequence of PTs $\beta \rightarrow>0$ and $\beta \rightarrow \alpha$ are exhibited in diagrams in Fig.5, constructed by pole figures of line broadening for the corresponding phases.

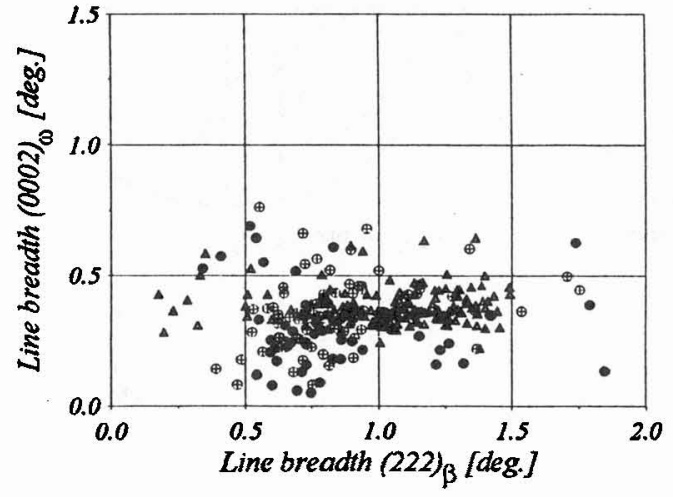

a)

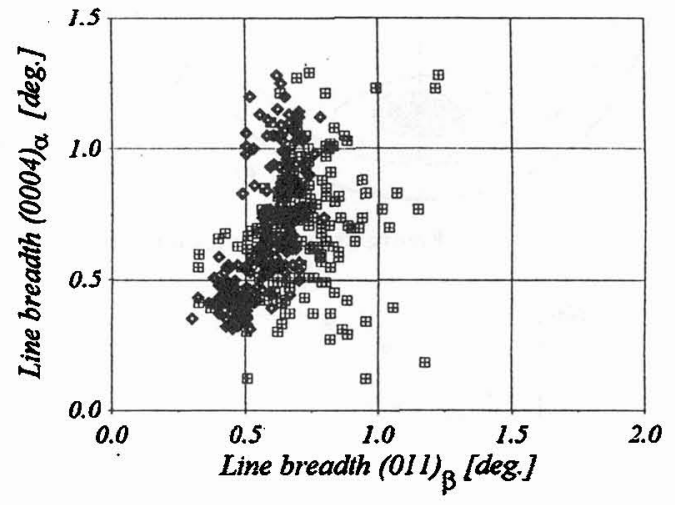

b)

Figure 5: Corrclation betwecn: a) WPF(0002) $)_{\omega}$ and $W P F(222)_{\beta} ;$ b) $W P F(0004)_{\alpha}$ and $W P F(011)_{\beta}$.

In order to check the validity of the preliminary hypotheses about influence of residual deformation

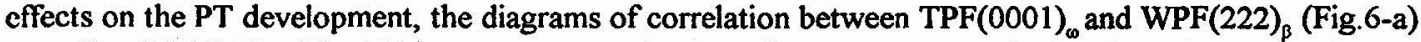
as well as $\mathrm{PPF}(222)_{\beta}$ (Fig.6-b) were constructed. These diagrams connect the fraction of $\omega$-grains, having considered orientations of normals $\langle 0001\rangle$, with the lattice condition in original $\beta$-grains along normals $\langle 111\rangle$, having the corresponding orientations. The correlation between $\operatorname{PPF}(0002)_{\omega}$ and $\operatorname{PPF}(222)_{\beta}$ is depicted by the diagram in Fig.7, which allows to compare the relative scattering of interplanar spacing for the original $\beta$-phase and the derivative $\omega$-phase.

\section{DISCUSSION}

The obtained data on PT $\beta \rightarrow \infty$ and their comparison with the data on PT $\beta \rightarrow \alpha$ in the same alloy $\mathrm{Zr}-20 \% \mathrm{Nb}$ allow to reveal a number of tendencies: 


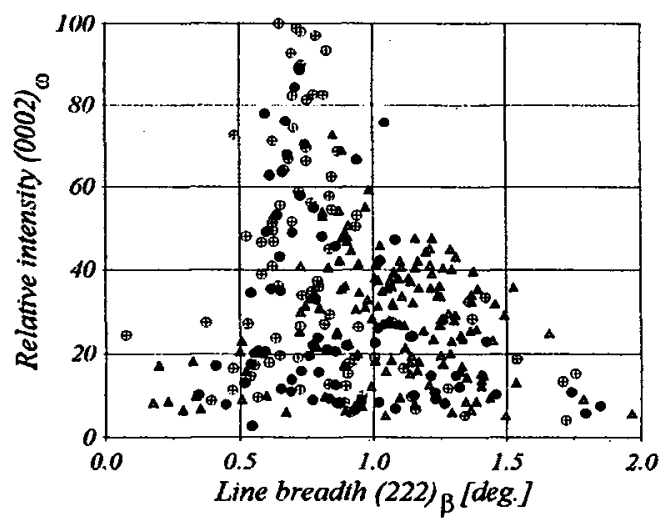

a)

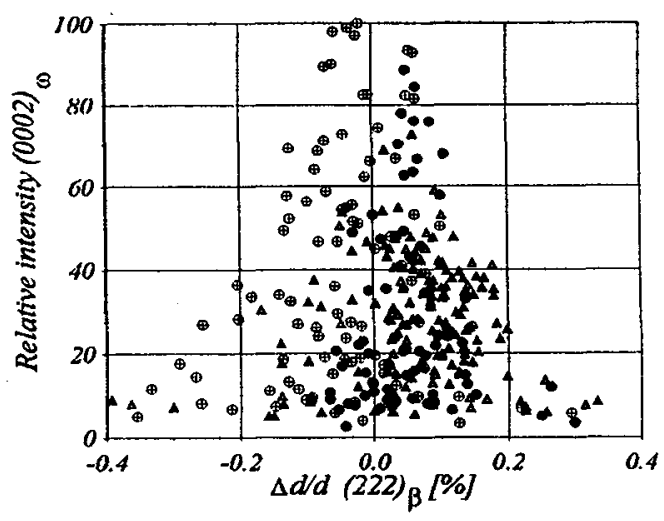

b)

Figure 6: Correlation between: a) $\operatorname{TPF}(0001)_{\omega}$ and $\mathrm{WPF}(222)_{\beta} ;$ b) $\operatorname{TPF}(0001)_{\omega}$ and $\operatorname{PPF}(222)_{\beta}$.

1) Pole figures and correlation diagrams for $\omega$-phase do not change as the annealing duration increases, whereas pole figures for $\alpha$-phase show a significant growth of texture maxima depending on the annealing duration. Hence, similar to martensitic transformations, the diffusionless $P T \quad \beta->\omega$ is athermal, whereas the diffusion PT $\beta \rightarrow \alpha$ is isothermal.

2) The $\omega$-phase forms in all $\beta$-grains, no matter how dispersed these grains and how distorted their lattice may be. Moreover, PT $\beta \rightarrow \omega$ is somewhat more active in texture minima, where both grain dispersion and lattice distortion are maximal (Fig.3-a). On the contrary, PT $\beta \rightarrow \alpha$ becomes noticeable only in those regions of TPF, where the relative intensity as well as the degree of grain dispersion are sufficiently high (Fig.3-b). This difference becomes understandable, when taking into account that PT $\beta \rightarrow \omega$ can embrace in principle the whole of an initial $\beta-\mathrm{Zr}$ grain, whereas formation of $\alpha$-phase, according to the $\mathrm{Zr}-\mathrm{Nb}$ phase diagram [7], develops with the necessary release of the residual $\beta-\mathrm{Nb}$ phase and, as a result, is accompanied by formation of new interphase boundaries within initial $\beta$-grains. Therefore, in smallest $\beta$-grains

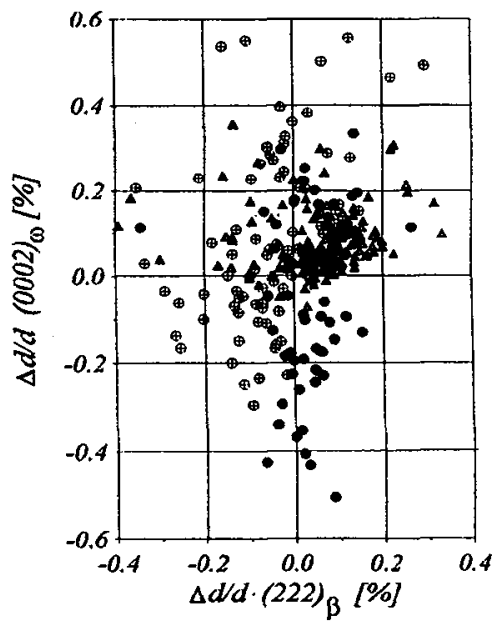

Figure 7: Correlation between $\mathrm{PPF}(0002)_{\infty}$ and $\mathrm{PPF}(222)_{\beta}$ PT $\beta \rightarrow \alpha$ can occur to be energetically unprofitable.

3) While grains of the original $\beta$-phase differ significantly in lattice condition .estimated by physical

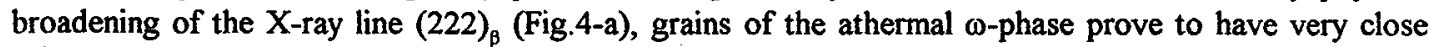
substructure parameters, as it is seen by small scattering of half-width values for the $X$-ray line $(0002)_{\omega}$ in Fig.4-b and 5-a. As a result of PT $\beta \rightarrow \omega$, the level of physical line broadening decreases, i.e. $\omega$-particles are coarser than coherent domains in the initial $\beta$-phase and/or have the more perfect lattice, than the majority of $\beta$-grains. This contradicts to the widespread idea that the $\omega$-phase is very dispersed. In the case of PT $\beta \rightarrow \alpha$ the opposite tendency takes place (Fig.5-b), involving the distinct interconnection of lattice conditions for original and derivative phases as well as their additional scattering. Since $\alpha$-grains form by decomposition of the quenched initial phase into two equilibrium phases, the final structure of the monotectoid alloy is characterized by increased dispersity of components.

4) As it is usual for rolled BCC metals [1] and can be seen in Fig.4-a and 6-a, $\beta$-grains with the rolling plane (111) are more dispersed and have more distorted lattice, than grains of the main texture component $\{001\}<011>$. But $\omega$-grains, descended from $\beta$-grains with the rolling plane (111), exhibit the same value 
of line broadening as others. At the same time, both $\omega$ - and $\alpha$-phases form in these grains with some advance relative to $\beta$-grains with other orientations.

5) Both transformations of the BCC phase into phases with hexagonal lattices are accompanied by essential redistribution of normals interconnected by the orientation relationships. So, in TPF $\{111\}_{\beta}$ maximal values of pole density take place within texture maxima at the diameter RD-RD, but in TPF(0001), maxima at the diameter TD-TD become the highest already (Fig.3-a). Hence, elementary prisms of the $\omega$-lattice grow preferably on those planes $\{111\}_{\beta}$, which have projections at the diameter TD-TD. This is because of the anisotropic character of residual elastic microstresses acting in $\beta$-grains. Fig.6-b shows that along normals $<111\rangle_{\beta}$ close to the diameter RD-RD the lattice of the quenched rolled $\beta$-phase was elastically extended, while in the same grains along normals $\langle 111\rangle_{\beta}$ close to the diameter TD-TD it was elastically compressed. It follows herefrom that the elastic compression promotes PT $\beta->\omega$, whereas the elastic extension hinders it to some degree.

6) In consequence of PT $\beta->\omega$ the local elastic deformation of crystalline lattice $\Delta d / d$ increases for the majority of points in the orientational space (Fig.7). The $\Delta d / d$ distribution in PPF (0002) (Fig.2-c) exhibits a very distinct cross-wise arrangement of maxima and minima, providing an equilibrium of elastic stresses of 2 nd kind with reference to the symmetry planes of the preceding rolling process. According to [2], such an arrangement is typical for rolled materials, but in the given case the $\omega$-phase, formed by annealing of the rolled $\beta$-phase, retains and amplifies this feature, showing thereby a peculiar heredity of substructure inhomogeneity.

\section{SUMMARY}

The presented experimental results demonstrate efficiency of the used method to study phase transformations, when the PT process is split into subprocesses developing in grains and/or along axes with different orientations. As applied to PTs $\beta \rightarrow \infty$ and $\beta->\alpha$ in the cold-rolled quenched alloy $\mathrm{Zr}-20 \% \mathrm{Nb}$, a number of their characteristic features was revealed, including modes of resulting changes in lattice condition, the influence of tensile and compressive residual elastic stresses on the orientation of new $\omega$-grains, some specific manifestations of heredity of substructure inhomogeneity.

\section{References}

[1] Perlovich Yu., "Development of strain hardening inhomogeneity during texture formation under rolling of bcc-metals", Numerical Prediction of Deformation Processes and the Behaviour of Real Materials, 15th Riso International Symposium on Material Science, 5-9 September 1994, S.A.Andersen et al. Eds. (Riso National Laboratory, Roskilde, Denmark, 1994) pp.445-450.

[2] Perlovich Yu., Bunge H.J., Isaenkova M. Textures and Microstructures 29 (1997) (in the press).

[3] Wcislak L., Bunge H.J. Texture Analysis with a Position Sensitive Detector (Cuvilier Verlag, Göttingen, 1996).

[4] Perlovich Yu., Bunge H.J., Fesenko V., Isaenkova M., Park N.J., Wcislak L., Zuev M. J.Phys. IV France, Cl 6 (1996) 149-156.

[5] Perlovich Yu., Bunge H.J., Fesenko V., Isaenkova M., "X-ray study of structure inhomogeneity in textured materials", Textures of Materials, 11 th International Conference on Textures of Materials, Xian, China, 1996, Z.Liang et al. Eds. (International Academic Publishers, 1996) pp.1455-1460.

[6] Taylor A., X-Ray Metallography (John Wiley \& Sons, Inc., New York - London, 1961).

[7] Douglass D.L., The Metallurgy of Zirconium (International Atomic Energy Agency, Viena, 1971) pp.13-23, 187-203.

[8] Hatt B.A., Roberts J.A. Acta metall. 8 (1960) 575-584. 